\title{
COHOMOLOGICAL INVARIANTS FOR ORTHOGONAL INVOLUTIONS ON DEGREE 8 ALGEBRAS
}

\author{
ANNE QUÉGUINER-MATHIEU AND JEAN-PIERRE TIGNOL
}

\begin{abstract}
Using triality, we define a relative Arason invariant for orthogonal involutions on a -possibly division- central simple algebra of degree 8. This invariant detects hyperbolicity, but it does not detect isomorphism. We produce explicit examples, in index 4 and 8 , of non isomorphic involutions with trivial relative Arason invariant.
\end{abstract}

The discriminant and the Clifford algebra are classical invariants of quadratic forms over a field $F$ of characteristic different from 2. Up to similarity, the discriminant classifies quadratic forms of dimension 2, while the even part of the Clifford algebra classifies forms of dimension 4. In [2], Arason defined an invariant $e_{3}$, for even-dimensional quadratic forms with trivial discriminant and split Clifford algebra, which has values in $H^{3}\left(F, \mu_{2}\right)$. Again, this invariant is classifying in dimension 8 , that is for forms similar to a 3 -fold Pfister form. The main purpose of this paper is to study the same question for orthogonal involutions on central simple algebras of degree 8 .

To see the relation between quadratic forms and involutions, recall that every (nondegenerate) quadratic form $q$ on an $F$-vector space $V$ defines an adjoint involution $\operatorname{ad}_{q}$ on the endomorphism algebra $\operatorname{End}_{F} V$, and every orthogonal involution on $\operatorname{End}_{F} V$ has the form $\operatorname{ad}_{q}$ for some (nondegenerate) quadratic form $q$ on $V$, uniquely determined up to a scalar factor, see [18, p. 1]. Therefore, orthogonal involutions on central simple algebras can be viewed as twisted forms (in the sense of Galois cohomology) of quadratic forms up to scalars. Analogues for orthogonal involutions of the discriminant and the (even) Clifford algebra were defined by Jacobson and Tits. By [18, (7.4) and $\S 15 . \mathrm{B}]$, the discriminant classifies orthogonal involutions on a given quaternion algebra, and the Clifford algebra classifies orthogonal involutions on a given biquaternion algebra.

On the other hand, it was shown in $[4, \S 3.4]$ that the Arason invariant does not extend to orthogonal involutions if the underlying algebra is a degree 8 division algebra. In this paper, we define a relative Arason invariant for orthogonal involutions on a degree 8 (possibly division) central simple algebra $A$ over $F$. Our construction, based on triality $[18, \S 42]$, is specific to this degree. Namely, we assign to any pair of involutions $\left(\sigma, \sigma^{\prime}\right)$ on $A$ with trivial discriminant and Clifford invariant, a degree 3 cohomology class $e_{3}\left(\sigma / \sigma^{\prime}\right) \in H^{3}\left(F, \mu_{2}\right) / F^{\times} \cdot[A]$, explicitly described as the Arason invariant of some quadratic form associated to $\left(\sigma, \sigma^{\prime}\right)$, and uniquely defined up to a multiple of the Brauer class of $A$ (see 1.4). This invariant commutes with scalar extensions, and is compatible with the Arason invariant for

Date: December 17, 2010.

Both authors acknowledge support from Wallonie-Bruxelles International and the French government in the framework of "Partenariats Hubert Currien". 
quadratic forms, meaning that if $A$ is split, and the involutions $\sigma$ and $\sigma^{\prime}$ are respectively adjoint to the 3 -fold Pfister forms $\pi$ and $\pi^{\prime}$, then $e_{3}\left(\sigma / \sigma^{\prime}\right)=e_{3}(\pi)-e_{3}\left(\pi^{\prime}\right)$ (see 1.5).

If the algebra $A$ is endowed with an involution $\sigma$ with trivial discriminant and Clifford invariant, it is isomorphic to a tensor product of three quaternion algebras by $[18,(42.11)]$. Hence, combining [23] and [17, 4.6], we get that the restriction map induces an injection

$$
H^{3}(F, \mathbb{Q} / \mathbb{Z}(2)) / F^{\times} \cdot[A] \rightarrow H^{3}\left(F_{A}, \mathbb{Q} / \mathbb{Z}(2)\right),
$$

where $F_{A}$ is a generic splitting field of $A$. Hence, the relative Arason invariant $e_{3}\left(\sigma / \sigma^{\prime}\right)$ is uniquely determined by its prescribed value in the split case. In particular, it coincides with the relative Arason invariant that one may define using the Rost invariant for Spin groups (see $[11, \S 3]$ and $[31, \S 3.5]$ ). The definition based on the Rost invariant, as opposed to the one proposed here, is also valid in higher degree. The resulting invariant is studied in [26], where we provide a formula for computing it in various cases. This invariant is not anymore uniquely determined by its value in the split case (see [11, Cor. 10.2]). It has values in $H^{3}\left(F, \mu_{4}^{\otimes 2}\right) / F^{\times} \cdot[A]$, and need not in general be represented by a class of $H^{3}\left(F, \mu_{2}\right)$ (see [26]).

Coming back to the present paper, note that in degree 8 , contrary to what happens for quadratic forms, the relative $e_{3}$-invariant is not classifying. Theorem 2.1 below states that the involutions $\sigma$ and $\sigma^{\prime}$ have trivial relative $e_{3}$ if and only if they are conjugate over any splitting field of $A$. As was noticed by Sivatski [27, Prop. 4], Hoffmann's examples of non-similar and half-neighbours 8-dimensional quadratic forms (see $[12, \S 4]$ ) prove the existence of non-isomorphic involutions that become isomorphic over any splitting field of the algebra. Using this, we produce in $\S 3$ explicit examples of non-conjugate involutions $\sigma$ and $\sigma^{\prime}$, with $e_{3}\left(\sigma / \sigma^{\prime}\right)=0$, on algebras that have index 4 and 8 .

To classify involutions in degree 8 , we define in 2.5 an additional relative invariant $e_{4}\left(\sigma / \sigma^{\prime}\right)$, associated to any pair of involutions $\left(\sigma, \sigma^{\prime}\right)$ with $e_{3}\left(\sigma / \sigma^{\prime}\right)=0$, and which has values in a quotient of $H^{4}\left(F, \mu_{2}\right)$. We prove that the totally decomposable involutions $\sigma$ and $\sigma^{\prime}$ of $A$ are isomorphic if and only if the relative invariants $e_{3}\left(\sigma / \sigma^{\prime}\right)$ and $e_{4}\left(\sigma / \sigma^{\prime}\right)$ are both trivial.

Even though the relative Arason invariant does not detect isomorphism, it detects hyperbolicity. This is explained in $\S 4$, where we concentrate on algebras of index $\leq 4$. As in Garibaldi $[11, \S 3]$, we define the Arason invariant of a totally decomposable involution $\sigma$ to be its relative Arason invariant with respect to an arbitrary hyperbolic involution, and we prove it vanishes if and only if the involution $\sigma$ is hyperbolic. We also provide explicit formulas for computing this invariant in terms of some decomposition of $(A, \sigma)$, and give necessary and sufficient conditions under which it is represented by a single symbol of $H^{3}\left(F, \mu_{2}\right)$ (see 4.3 and 4.8).

Notation. Throughout this paper, we work over a base field $F$ of characteristic different from 2. We refer to [18] and to [20] for background information on central simple algebras with involution and on quadratic forms.

The invariants defined below are represented by cohomology classes of order 2 . Hence, for every integer $n \geq 0$, we use the notation

$$
H^{n}(F)=H^{n}\left(\operatorname{Gal}\left(F_{\text {sep }} / F\right), \mu_{2}\right) .
$$


In particular, $H^{1}(F)=F^{\times} / F^{\times 2}$, and $H^{2}(F)=\operatorname{Br}_{2}(F)$ is the 2-part of the Brauer group of $F$. For every $a \in F^{\times}$we let $(a) \in H^{1}(F)$ be the square class of $a$, and for $a_{1}, \ldots, a_{n} \in F^{\times}$we let $\left(a_{1}, \ldots, a_{n}\right) \in H^{n}(F)$ be the cup-product

$$
\left(a_{1}, \ldots, a_{n}\right)=\left(a_{1}\right) \cdot \cdots \cdot\left(a_{n}\right) .
$$

In particular, $\left(a_{1}, a_{2}\right)$ denotes the Brauer class of the quaternion algebra $\left(a_{1}, a_{2}\right)_{F}$. In general, if $A$ is an $F$-central simple algebra, we let $[A]$ be the Brauer class of $A$. If $A$ has exponent 2, we view [A] as an element in $H^{2}(F)$. This applies in particular to the Clifford algebra of any even-dimensional quadratic form.

If $L$ is a field extension of $F$, we let $A_{L}=A \otimes_{F} L$ be the $L$-algebra obtained from $A$ by extending scalars. On the other hand, $N_{L / F}$ denotes the corestriction map from $H^{n}(L)$ to $H^{n}(F)$. If $A$ has exponent 2, the notation $M_{A}^{3}(L)$ stands for the quotient of $H^{3}(L)$ defined by

$$
M_{A}^{3}(L)=H^{3}(L) /\left(L^{\times} \cdot\left[A_{L}\right]\right),
$$

where $L^{\times} \cdot\left[A_{L}\right]$ denotes the subgroup $\left\{(\ell) \cdot\left[A_{L}\right], \ell \in L^{\times}\right\} \subset H^{3}(L)$. Let $F_{A}$ be the function field of the Severi-Brauer variety of $A$, which is a generic splitting field of $A$. Scalar extension from $F$ to $F_{A}$ yields a group homomorphism

$$
M_{A}^{3}(F) \rightarrow M_{A}^{3}\left(F_{A}\right)=H^{3}\left(F_{A}\right) .
$$

It follows from [23] and [17, 4.5 and 4.6] that this map is injective when $A$ decomposes into a tensor product of three quaternion algebras, even though it is not injective in general, even in degree 8.

We depart from the notation in $[20]$ by letting $\left\langle\left\langle a_{1}, \ldots, a_{n}\right\rangle\right\rangle$ denote the $n$-fold Pfister form

$$
\left\langle\left\langle a_{1}, \ldots, a_{n}\right\rangle\right\rangle=\left\langle 1,-a_{1}\right\rangle \cdot \ldots \cdot\left\langle 1,-a_{n}\right\rangle \quad \text { for } a_{1}, \ldots, a_{n} \in F^{\times} .
$$

For any quadratic form $q$ over $F$, we let $d(q) \in F^{\times} / F^{\times 2}$ be the (signed) discriminant of $q, \mathcal{C}(q)$ its Clifford algebra, and $\mathcal{C}_{0}(q)$ the even part of $\mathcal{C}(q)$. If $q$ has evendimension, its Clifford invariant is the Brauer class of $\mathcal{C}(q)$. If additionally it has trivial discriminant, then $\mathcal{C}_{0}(q)$ is a direct product of two central simple algebras over $F$, which are Brauer equivalent to $\mathcal{C}(q)$. An 8-dimensional quadratic form has trivial discriminant and Clifford invariant if and only if it is similar to a 3 -fold Pfister form. If so, its Arason invariant is given, with our conventions, by

$$
e_{3}\left(\left\langle\left\langle a_{1}, a_{2}, a_{3}\right\rangle\right\rangle\right)=\left(a_{1}, a_{2}, a_{3}\right) .
$$

More generally, for any quadratic form $q$ of even dimension and trivial discriminant, and any scalar $a \in F^{\times}$, the quadratic form $\langle\langle a\rangle\rangle \otimes q$ has trivial discriminant and Clifford invariant. Its Arason invariant is given by

$$
e_{3}(\langle\langle a\rangle\rangle \otimes q)=(a) \cdot[\mathcal{C}(q)] .
$$

For any orthogonal involution $\sigma$ on $A$, we let $d(\sigma) \in F^{\times} / F^{\times 2}$ be its (signed) discriminant and $\mathcal{C}(A, \sigma)$ its Clifford algebra, as defined in [18, $\S 7$ and $\S 8]$. If $q$ is an even-dimensional quadratic form on the $F$-vector space $V$, we denote by $\mathrm{Ad}_{q}$ the split algebra with involution $\left(\operatorname{End}_{F}(V), \operatorname{ad}_{q}\right)$. It satisfies

$$
d\left(\operatorname{ad}_{q}\right)=d(q) \quad \text { and } \quad \mathcal{C}\left(\operatorname{Ad}_{q}\right)=\mathcal{C}_{0}(q)
$$

Any involution $\sigma$ with trivial discriminant has Clifford algebra isomorphic to a direct product of two $F$-central simple algebras

$$
\mathcal{C}(A, \sigma)=\mathcal{C}^{+}(A, \sigma) \times \mathcal{C}^{-}(A, \sigma) .
$$


If moreover the degree of $A$ is divisible by 4 , then the Brauer classes of $\mathcal{C}^{ \pm}(A, \sigma)$ have order 2 and satisfy

$$
\left[\mathcal{C}^{+}(A, \sigma)\right]+\left[\mathcal{C}^{-}(A, \sigma)\right]=[A]
$$

We say that $\sigma$ has trivial Clifford invariant if one of the two components is split, so that the other is Brauer equivalent to $A$; in the split case, this amounts to $[\mathcal{C}(q)]=0$.

If $A$ has degree 4, and $\sigma$ has trivial discriminant, then $\mathcal{C}(A, \sigma)$ is a direct product $Q_{1} \times Q_{2}$ of two quaternion algebras $Q_{1}$ and $Q_{2}$ over $F$. Moreover, by [18, (15.12)], we have

$$
(A, \sigma)=\left(Q_{1},-\right) \otimes\left(Q_{2},-\right)
$$

where - stands for canonical involutions. Note that such an $(A, \sigma)$ can also be written as a product of two quaternion algebras with orthogonal involutions. For instance, the same computation as in [25, Proof of Lem. 6.1] shows that for any $a, a^{\prime}, \delta, \delta^{\prime} \in F^{\times}$,

$$
\left((a, \delta)_{F}, \rho\right) \otimes\left(\left(a^{\prime}, \delta^{\prime}\right)_{F}, \rho^{\prime}\right) \cong\left(\left(a \delta^{\prime}, \delta\right)_{F},-\right) \otimes\left(\left(a^{\prime} \delta, \delta^{\prime}\right)_{F},-\right)
$$

where $\rho$ and $\rho^{\prime}$ are orthogonal involutions of discriminant $(\delta)$ and $\left(\delta^{\prime}\right)$ respectively.

\section{Relative Arason invariant}

Throughout the paper, $A$ denotes a central simple $F$-algebra of degree 8 . We assume it is endowed with an orthogonal involution $\sigma$ that has trivial discriminant and Clifford invariant. By $[18,(42.11)]$, this precisely means that $(A, \sigma)$ is totally decomposable, i.e. isomorphic to a tensor product of three quaternion algebras with involution. By the proof of $[18,(42.11)]$ we actually have:

Lemma 1.1. There exists a quadratic form $\varphi$ over $F$, unique up to similarity, such that

$$
\left(\mathcal{C}_{0}(\varphi), \operatorname{can}\right)=(A, \sigma) \times(A, \sigma)
$$

We call this form $\varphi$ the quadratic form associated to $(A, \sigma)$ by triality.

Indeed, since $A$ has degree 8 , the canonical involution on $\mathcal{C}(A, \sigma)$ induces an orthogonal involution on each factor; the involution on the split factor is adjoint to a quadratic form $\varphi$. Now, it follows from triality $[18,(42.3)]$ (see also [25, $5.1])$ that the even Clifford algebra of $\varphi$ with canonical involution is $\left(\mathcal{C}_{0}(\varphi)\right.$, can) $=$ $(A, \sigma) \times(A, \sigma)$. Moreover, for any 8-dimensional form $\psi$ with even Clifford algebra $\left(C_{0}(\psi)\right.$, can $)=(A, \sigma) \times(A, \sigma)$, we have again by triality, $(\mathcal{C}(A, \sigma), \underline{\sigma})=$ $\operatorname{Ad}_{\psi} \times(A, \sigma)$. Hence $\operatorname{Ad}_{\psi}$ is isomorphic to $\operatorname{Ad}_{\varphi}$, so that $\psi$ is similar to $\varphi$.

Example 1.2. Consider the algebra with involution

$$
(A, \sigma)=\left(Q_{1} \otimes Q_{2} \otimes Q_{3},-\otimes-\otimes \rho\right),
$$

where $Q_{i}$, for $i \in\{1,2,3\}$, is an $F$-quaternion algebra, - stands for canonical involutions, and $\rho$ is an orthogonal involution of $Q_{3}$ of discriminant $d(\rho)=(\delta)$. We may then find $\gamma \in F^{\times}$such that $\left[Q_{3}\right]=(\delta, \gamma)$. Note that we also have $Q_{3} \cong$ $(\gamma,-\gamma \delta)_{F}$ and the anticommuting pure quaternions of square $\gamma$ and $-\gamma \delta$ are skewsymmetric under $\rho$. Let $\left[Q_{1}\right]=(\alpha, \beta)$ and $\left[Q_{2}\right]=(\varepsilon, \zeta)$. We claim that the following quadratic form is associated to $(A, \sigma)$ by triality:

$$
\varphi=\langle 1,-\alpha,-\beta, \alpha \beta \gamma,-\alpha \beta \gamma \delta, \alpha \beta \delta \varepsilon, \alpha \beta \delta \zeta,-\alpha \beta \delta \varepsilon \zeta\rangle .
$$


To see this, let $\left(e_{i}\right)_{1 \leq i \leq 8}$ be an orthogonal basis for $\varphi$ corresponding to this diagonalisation. One may check that the elements $i_{1}=e_{1} e_{2}, j_{1}=e_{1} e_{3}, i_{2}=e_{1} e_{2} e_{3} e_{4}$, $j_{2}=e_{1} e_{2} e_{3} e_{5}, i_{3}=e_{1} e_{2} e_{3} e_{4} e_{5} e_{6}, j_{3}=e_{1} e_{2} e_{3} e_{4} e_{5} e_{7}$ and $z=e_{1} e_{2} e_{3} e_{4} e_{5} e_{6} e_{7} e_{8}$ of $\mathcal{C}_{0}(\varphi)$ generate an algebra with involution isomorphic to

$$
(\alpha, \beta)_{F} \otimes(\gamma,-\gamma \delta)_{F} \otimes(\varepsilon, \zeta)_{F} \otimes(F \times F)
$$

endowed with the involution $-\otimes \rho \otimes-\otimes \mathrm{Id}$, which is nothing but $(A, \sigma) \times(A, \sigma)$. Hence, by dimension count, $\left(\mathcal{C}_{0}(\varphi)\right.$, can $) \cong(A, \sigma) \times(A, \sigma)$.

Consider now another totally decomposable involution $\sigma^{\prime}$ on $A$, and denote by $\varphi^{\prime}$ the quadratic form associated to $\sigma^{\prime}$ by triality. Since $\mathcal{C}(\varphi)$ and $\mathcal{C}\left(\varphi^{\prime}\right)$ are both Brauer-equivalent to $A$, the difference $\varphi-\varphi^{\prime}$ belongs to $I^{3} F$. The forms $\varphi$ and $\varphi^{\prime}$ are uniquely defined only up to a scalar factor. The following lemma shows that the class in $M_{A}^{3}(F)$ of the Arason invariant of $\varphi-\varphi^{\prime}$ does not depend on these scalars:

Lemma 1.3. For any $\lambda, \lambda^{\prime} \in F^{\times}$, we have

$$
e_{3}\left(\langle\lambda\rangle \varphi-\left\langle\lambda^{\prime}\right\rangle \varphi^{\prime}\right)=e_{3}\left(\varphi-\varphi^{\prime}\right) \in M_{A}^{3}(F) .
$$

Proof. In the Witt ring $W F$ of the field $F$, we have

$$
\langle\lambda\rangle \varphi-\left\langle\lambda^{\prime}\right\rangle \varphi^{\prime}=\left(\varphi-\varphi^{\prime}\right)-\langle\langle\lambda\rangle\rangle \varphi+\left\langle\left\langle\lambda^{\prime}\right\rangle\right\rangle \varphi^{\prime} .
$$

Since $\varphi, \varphi^{\prime}$ both have trivial discriminant and Clifford algebra Brauer equivalent to $A$, each term of the sum on the right side has trivial discriminant and Clifford invariant. Hence we get

$$
e_{3}\left(\langle\lambda\rangle \varphi-\left\langle\lambda^{\prime}\right\rangle \varphi^{\prime}\right)=e_{3}\left(\varphi-\varphi^{\prime}\right)-(\lambda) \cdot[A]+\left(\lambda^{\prime}\right) \cdot[A],
$$

and the lemma is proved.

This leads to the following definition of a relative Arason invariant for totally decomposable involutions:

Definition 1.4. Let $A$ be a degree 8 algebra endowed with two totally decomposable involutions $\sigma$ and $\sigma^{\prime}$. The Arason invariant of $\sigma$ with respect to $\sigma^{\prime}$ is

$$
e_{3}\left(\sigma / \sigma^{\prime}\right)=e_{3}\left(\varphi-\varphi^{\prime}\right) \in M_{A}^{3}(F),
$$

where $\varphi$ and $\varphi^{\prime}$ are quadratic forms respectively associated to $\sigma$ and $\sigma^{\prime}$ by triality as in Lemma 1.1 .

This is a well defined invariant of the pair of involutions $\left(\sigma, \sigma^{\prime}\right)$ by the previous lemma. It is functorial, since the Clifford algebra construction and the Arason invariant for quadratic forms commute with scalar extensions. Moreover, it readily follows from the definition that $e_{3}\left(\sigma^{\prime} / \sigma\right)=e_{3}\left(\sigma / \sigma^{\prime}\right)$, and if $\sigma^{\prime \prime}$ is another totally decomposable involution on $A$, we have

$$
e_{3}\left(\sigma / \sigma^{\prime \prime}\right)=e_{3}\left(\sigma / \sigma^{\prime}\right)+e_{3}\left(\sigma^{\prime} / \sigma^{\prime \prime}\right)
$$

Let us now compute its value in the split case:

Proposition 1.5. If $A$ is split, and $\sigma$ and $\sigma^{\prime}$ are respectively adjoint to the threefold Pfister forms $\pi$ and $\pi^{\prime}$, then

$$
e_{3}\left(\sigma / \sigma^{\prime}\right)=e_{3}(\pi)-e_{3}\left(\pi^{\prime}\right) \in H^{3}(F) .
$$


Indeed, as explained in $[25,5.1]$, the algebra $A$ is split if and only if the forms $\varphi$ and $\varphi^{\prime}$ are similar to 3-fold Pfister forms $\pi$ and $\pi^{\prime}$, and we then have $\sigma=\operatorname{ad}_{\pi}$ and $\sigma^{\prime}=\operatorname{ad}_{\pi^{\prime}}$. Hence we get $e_{3}\left(\sigma / \sigma^{\prime}\right)=e_{3}\left(\varphi-\varphi^{\prime}\right)=e_{3}(\pi)-e_{3}\left(\pi^{\prime}\right) \in H^{3}(F)$.

Remark 1.6. Let $A$ be an algebra of exponent 2 and $\varphi$ a quadratic form of even dimension and trivial discriminant such that $\mathcal{C}(\varphi)$ is Brauer equivalent to $A$. In [16], Bruno Kahn explains how one can view $e_{3}\left(\varphi_{F_{A}}\right) \in H^{3}\left(F_{A}\right) / H^{3}(F)$ as an invariant of the algebra $A$. In degree 8 , we may choose $\varphi$ of dimension 8 , which amounts to the choice of a totally decomposable orthogonal involution of $A$. For two different choices $\varphi$ and $\varphi^{\prime}$, the relative Arason invariant of the corresponding involutions defined above precisely is the part that is factored out in Bruno Kahn's invariant.

Example 1.7. Let us consider a tensor product of three quaternion algebras, $A=$ $Q_{1} \otimes Q_{2} \otimes Q_{3}$ endowed with the involutions $\sigma=-\otimes-\otimes \rho$ and $\sigma^{\prime}=-\otimes-\otimes \rho^{\prime}$, where - stands for canonical involutions, and $\rho$ and $\rho^{\prime}$ are orthogonal involutions of $Q_{3}$ of discriminant respectively equal to $(\delta)$ and $\left(\delta^{\prime}\right)$. By the common slot lemma [2, Lemma 1.7], there exists $\gamma \in F^{\times}$such that $\left[Q_{3}\right]=(\delta, \gamma)=\left(\delta^{\prime}, \gamma\right)$. Since $\left(\delta \delta^{\prime}, \gamma\right)=0 \in H^{2}(F)$, we have $\left(\delta \delta^{\prime}\right) \cdot[A]=\left(\delta \delta^{\prime}\right) \cdot\left[Q_{1}\right]+\left(\delta \delta^{\prime}\right) \cdot\left[Q_{2}\right]$, hence, $\left(\delta \delta^{\prime}\right) \cdot\left[Q_{1}\right] \equiv\left(\delta \delta^{\prime}\right) \cdot\left[Q_{2}\right] \bmod F^{\times} \cdot[A]$. We claim that the relative Arason invariant is given by

$$
e_{3}\left(\sigma / \sigma^{\prime}\right)=\left(\delta \delta^{\prime}\right) \cdot\left[Q_{1}\right]=\left(\delta \delta^{\prime}\right) \cdot\left[Q_{2}\right] \in M_{A}^{3}(F) .
$$

The proof goes as follows. Denote $\left[Q_{1}\right]=(\alpha, \beta)$ and $\left[Q_{2}\right]=(\varepsilon, \zeta)$. By Example 1.2, the following quadratic forms are associated respectively to $\sigma$ and to $\sigma^{\prime}$ by triality:

$$
\begin{array}{r}
\varphi=\langle 1,-\alpha,-\beta, \alpha \beta \gamma,-\alpha \beta \gamma \delta, \alpha \beta \delta \varepsilon, \alpha \beta \delta \zeta,-\alpha \beta \delta \varepsilon \zeta\rangle \\
\varphi^{\prime}=\left\langle 1,-\alpha,-\beta, \alpha \beta \gamma,-\alpha \beta \gamma \delta^{\prime}, \alpha \beta \delta^{\prime} \varepsilon, \alpha \beta \delta^{\prime} \zeta,-\alpha \beta \delta^{\prime} \varepsilon \zeta\right\rangle .
\end{array}
$$

To compute $e_{3}\left(\varphi-\varphi^{\prime}\right)$, note that

$$
\varphi-\varphi^{\prime}=\langle-\alpha \beta \delta\rangle\left\langle\left\langle\delta \delta^{\prime}\right\rangle\right\rangle\langle\gamma,-\varepsilon,-\zeta, \varepsilon \zeta\rangle .
$$

Moreover, since $\left(\delta \delta^{\prime}, \gamma\right)=0, \gamma$ is a similarity factor for $\left\langle\left\langle\delta \delta^{\prime}\right\rangle\right\rangle$, and we get

$$
\varphi-\varphi^{\prime}=\langle-\alpha \beta \delta\rangle\left\langle\left\langle\delta \delta^{\prime}, \varepsilon, \zeta\right\rangle\right\rangle
$$

so that $e_{3}\left(\varphi-\varphi^{\prime}\right)=\left(\delta \delta^{\prime}, \varepsilon, \zeta\right)=\left(\delta \delta^{\prime}\right) \cdot\left[Q_{2}\right]$.

\section{THE $e_{4}$-INVARIANT}

As opposed to what happens for quadratic forms, the relative Arason invariant is not a complete invariant for totally decomposable involutions in degree 8. It only detects isomorphism after scalar extension to a splitting field of the algebra. More precisely, we have the following:

Theorem 2.1. Let $A$ be a degree 8 algebra endowed with two totally decomposable orthogonal involutions $\sigma$ and $\sigma^{\prime}$. We consider quadratic forms $\varphi$ and $\varphi^{\prime}$ respectively associated to $\sigma$ and $\sigma^{\prime}$ by triality as in Lemma 1.1. The following are equivalent:

(i) $e_{3}\left(\sigma / \sigma^{\prime}\right)=0 \in M_{A}^{3}(F)$;

(ii) $\sigma_{F_{A}} \cong \sigma_{F_{A}}^{\prime}$;

(iii) For any splitting field $L / F$ of the algebra $A$, we have $\sigma_{L} \cong \sigma_{L}^{\prime}$;

(iv) There exists $\mu \in F^{\times}$such that $\varphi+\langle\mu\rangle \varphi^{\prime}$ is similar to a 4-fold Pfister form, i.e. $\varphi$ and $\varphi^{\prime}$ are half-neighbours. 
Remark 2.2. Clearly, conditions (i) to (iv) imply that $\sigma$ and $\sigma^{\prime}$ are isomorphic if the algebra $A$ is split. From [19, Thm.3], this is still the case in index 2 (see $\S 4.1$ for a detailed proof).

Nevertheless, this is not true anymore in higher index. In $\S 3$ below we provide explicit examples of non isomorphic involutions having a trivial relative Arason invariant, mostly inspired from Hoffmann [12, §4]. The underlying algebra has index 4 or 8 .

Proof. The implication (i) $\Rightarrow$ (iii) follows from the observation that in the split case, $e_{3}\left(\sigma / \sigma^{\prime}\right)$ vanishes if and only if $\sigma$ and $\sigma^{\prime}$ are isomorphic, and (iii) $\Rightarrow$ (ii) is clear. If (ii) holds, then $e_{3}\left(\sigma / \sigma^{\prime}\right)_{F_{A}}=0$, hence $e_{3}\left(\sigma / \sigma^{\prime}\right)=0$ because, as explained in the introduction, the restriction map induces an injection

$$
M_{A}^{3}(F) \rightarrow H^{3}\left(F_{A}\right) .
$$

The equivalence of (ii) and (iv) is Sivatski's Proposition 4 [27], which is based on Laghribi [19]. Alternately, we may directly prove that (i) is equivalent to (iv) as follows. By definition, $e_{3}\left(\sigma / \sigma^{\prime}\right)=0$ if and only if there exists $\mu \in F^{\times}$such that

$$
e_{3}\left(\varphi-\varphi^{\prime}\right)=(\mu) \cdot[A] \text {. }
$$

Since $(\mu) \cdot[A]=e_{3}\left(\langle\langle\mu\rangle\rangle \varphi^{\prime}\right)$, this amounts to $e_{3}\left(\varphi-\langle\mu\rangle \varphi^{\prime}\right)=0$, i.e. $\varphi-\langle\mu\rangle \varphi^{\prime} \in I^{4}(F)$. The forms $\varphi$ and $\varphi^{\prime}$ both have dimension 8 , hence this exactly means that $\varphi-\langle\mu\rangle \varphi^{\prime}$ is similar to a 4 -fold Pfister form, and concludes the proof.

The quadratic forms $\varphi$ and $\varphi^{\prime}$ are only defined up to similarity, and the scalar $\mu$ need not be unique in general. Thus, we need the following computation to define an $e_{4}$-invariant:

Lemma 2.3. Consider two totally decomposable involutions $\sigma$ and $\sigma^{\prime}$ of $A$ with $e_{3}\left(\sigma / \sigma^{\prime}\right)=0$, and let $\varphi, \varphi^{\prime}$ and $\mu$ be as in Theorem 2.1. The subset

$$
E_{4}=\left\{e_{4}(\langle\langle\nu\rangle\rangle), \text { where } \nu \in F^{\times} \text {satisfies }(\nu) \cdot[A]=0\right\} \subset H^{4}(F)
$$

is a subgroup of $H^{4}(F)$. Moreover, for any $\lambda, \lambda^{\prime}$ such that $\langle\lambda\rangle \varphi-\left\langle\lambda^{\prime}\right\rangle \varphi^{\prime} \in I^{4}(F)$, the invariants $e_{4}\left(\varphi-\langle\mu\rangle \varphi^{\prime}\right)$ and $e_{4}\left(\langle\lambda\rangle \varphi-\left\langle\lambda^{\prime}\right\rangle \varphi^{\prime}\right)$ have the same image in $H^{4}(F) / E_{4}$.

Remark 2.4. Since $\varphi$ has trivial discriminant and Clifford algebra Brauer equivalent to $A$, the form $\langle\langle\nu\rangle\rangle \varphi$ is in $I^{4} F$ if and only if $(\nu) \cdot[A]=0 \in H^{3}(F)$. Moreover, the set $E_{4}$ does not depend on the choice of $\varphi$ in its similarity class, and since

$$
\varphi \equiv\langle\mu\rangle \varphi^{\prime} \bmod I^{4} F
$$

we also have

$$
E_{4}=\left\{e_{4}\left(\langle\langle\nu\rangle\rangle \varphi^{\prime}\right), \text { where } \nu \in F^{\times} \text {satisfies }(\nu) \cdot[A]=0\right\} .
$$

Proof. Consider $\nu$ and $\nu^{\prime} \in F^{\times}$satisfying $\langle\langle\nu\rangle\rangle \varphi I^{4} F$ and $\left\langle\left\langle\nu^{\prime}\right\rangle\right\rangle \varphi \in I^{4} F$. In the Witt group $W(F)$, we have

$$
\left\langle\left\langle\nu \nu^{\prime}\right\rangle\right\rangle \varphi=\langle\langle\nu\rangle\rangle \varphi+\langle\nu\rangle\left\langle\left\langle\nu^{\prime}\right\rangle\right\rangle \varphi .
$$

Hence $\left\langle\left\langle\nu \nu^{\prime}\right\rangle\right\rangle \varphi \in I^{4}(F)$ and

$$
e_{4}\left(\left\langle\left\langle\nu \nu^{\prime}\right\rangle\right\rangle \varphi\right)=e_{4}(\langle\langle\nu\rangle\rangle \varphi)+e_{4}\left(\left\langle\left\langle\nu^{\prime}\right\rangle\right\rangle \varphi\right) .
$$

This proves $E_{4}$ is a subgroup of $H^{4}(F)$.

Assume now that $\langle\lambda\rangle \varphi-\left\langle\lambda^{\prime}\right\rangle \varphi^{\prime} \in I^{4}(F)$. Since

$$
\langle\lambda\rangle \varphi-\left\langle\lambda^{\prime}\right\rangle \varphi^{\prime}=\langle\lambda\rangle\left\langle\left\langle\lambda \lambda^{\prime} \mu\right\rangle\right\rangle \varphi+\left\langle\mu \lambda^{\prime}\right\rangle\left(\varphi-\langle\mu\rangle \varphi^{\prime}\right),
$$


we get that $\left\langle\left\langle\lambda \lambda^{\prime} \mu\right\rangle\right\rangle \varphi \in I^{4} F$ and

$$
e_{4}\left(\langle\lambda\rangle \varphi-\left\langle\lambda^{\prime}\right\rangle \varphi^{\prime}\right) \equiv e_{4}\left(\varphi-\langle\mu\rangle \varphi^{\prime}\right) \bmod E_{4} .
$$

Hence, in the situation of Theorem 2.1, the image in $H^{4}(F) / E_{4}$ of $e_{4}\left(\varphi-\langle\mu\rangle \varphi^{\prime}\right)$ does not depend on the choice of the quadratic forms $\varphi$ and $\varphi^{\prime}$ associated to $\sigma$ and $\sigma^{\prime}$, nor on the factor $\mu$ such that $\varphi-\langle\mu\rangle \varphi^{\prime} \in I^{4} F$. This leads to the following definition:

Definition 2.5. Let $A$ be a degree 8 algebra, and $\sigma$ and $\sigma^{\prime}$ be two totally decomposable orthogonal involutions on $A$ such that $e_{3}\left(\sigma / \sigma^{\prime}\right)=0$. We let $\varphi$ and $\varphi^{\prime}$ be the quadratic forms respectively associated to $\sigma$ and $\sigma^{\prime}$ by triality. The relative $e_{4}$ invariant of $\sigma$ with respect to $\sigma^{\prime}$ is defined by

$$
e_{4}\left(\sigma / \sigma^{\prime}\right)=e_{4}\left(\varphi-\langle\mu\rangle \varphi^{\prime}\right) \in H^{4}(F) / E_{4}
$$

where $\mu$ is any scalar in $F^{\times}$satisfying $\varphi-\langle\mu\rangle \varphi^{\prime} \in I^{4}(F)$.

Again, this is a well defined and functorial invariant of the pair of involutions $\left(\sigma, \sigma^{\prime}\right)$ if $e_{3}\left(\sigma / \sigma^{\prime}\right)=0$. Moreover, we have:

Proposition 2.6. Let $A$ be a degree 8 algebra. Two orthogonal totally decomposable involutions $\sigma$ and $\sigma^{\prime}$ on $A$ are isomorphic if and only if

$$
e_{3}\left(\sigma / \sigma^{\prime}\right)=0 \quad \text { and } \quad e_{4}\left(\sigma / \sigma^{\prime}\right)=0 .
$$

Proof. The direct implication is clear. Assume conversely that $e_{3}\left(\sigma / \sigma^{\prime}\right)=0$ and $e_{4}\left(\sigma / \sigma^{\prime}\right)=0$. There exists $\nu \in F^{\times}$such that $\langle\langle\nu\rangle\rangle \varphi \in I^{4}(F)$ and

$$
e_{4}\left(\varphi-\langle\mu\rangle \varphi^{\prime}\right)=e_{4}(\langle\langle\nu\rangle\rangle \varphi) \in H^{4}(F)
$$

Hence, the form $\langle\nu\rangle \varphi-\langle\mu\rangle \varphi^{\prime}$ also is in $I^{4}(F)$ and has trivial $e_{4}$. Since it has dimension 16, it is hyperbolic, and we get that $\varphi$ and $\varphi^{\prime}$ are similar. By Lemma 1.1, this implies that $\sigma$ and $\sigma^{\prime}$ are isomorphic.

In the following statement, we use the $e_{4}$ invariant to show that some involutions are isomorphic. As opposed to this, we will use it in $\S 3$ to produce examples of non isomorphic involutions with trivial relative Arason invariant.

Proposition 2.7. Let $A$ be a degree 8 algebra, endowed with two orthogonal involutions $\sigma$ and $\sigma^{\prime}$. We assume that $A$ contains a product of two quaternion algebras $Q_{1} \otimes Q_{2}$, with split centralizer, which is stable under $\sigma$ and $\sigma^{\prime}$, and on which both involutions act as the product $-\otimes-$ of the canonical involutions of $Q_{1}$ and $Q_{2}$. If so, the involutions $\sigma$ and $\sigma^{\prime}$ are isomorphic if and only if $e_{3}\left(\sigma / \sigma^{\prime}\right)=0$.

Proof. The hypothesis means that $A$ decomposes as $A=Q_{1} \otimes Q_{2} \otimes M_{2}(F)$, and the involutions are given by $\sigma=-\otimes-\otimes$ ad $\langle\langle\delta\rangle\rangle$ and $\sigma^{\prime}=-\otimes-\otimes$ ad $\left\langle\left\langle\delta^{\prime}\right\rangle\right\rangle$, for some $\delta, \delta^{\prime} \in F^{\times}$. We let $n_{Q_{1}}=\langle\langle\alpha, \beta\rangle\rangle$ and $n_{Q_{2}}=\langle\langle\varepsilon, \zeta\rangle\rangle$ be the norm forms of $Q_{1}=$ $(\alpha, \beta)_{F}$ and $Q_{2}=(\varepsilon, \zeta)_{F}$. Since we are in the situation of Examples 1.2 and 1.7 with additionally $\gamma=1$, the forms $\varphi=n_{Q_{1}}+\langle-\alpha \beta \delta\rangle n_{Q_{2}}$ and $\varphi^{\prime}=n_{Q_{1}}+\left\langle-\alpha \beta \delta^{\prime}\right\rangle n_{Q_{2}}$ are respectively associated to $\sigma$ and $\sigma^{\prime}$ by triality, and $e_{3}\left(\sigma / \sigma^{\prime}\right)=\left(\delta \delta^{\prime}\right) \cdot\left[Q_{2}\right] \in M_{A}^{3}(F)$. To prove the result, it is enough to check that $e_{4}\left(\sigma / \sigma^{\prime}\right)$ vanishes as soon as $e_{3}\left(\sigma / \sigma^{\prime}\right)$ does.

Assume that $e_{3}\left(\sigma / \sigma^{\prime}\right)=0 \in M_{A}^{3}(F)$. There exists $\mu \in F^{\times}$such that $\left(\delta \delta^{\prime}\right) \cdot\left[Q_{2}\right]=$ $(\mu) \cdot[A]$, that is $\left(\delta \delta^{\prime} \mu\right) \cdot\left[Q_{2}\right]=(\mu) \cdot\left[Q_{1}\right]$. This means that the quadratic forms 
$\left\langle\left\langle\delta \delta^{\prime} \mu\right\rangle\right\rangle n_{Q_{2}}$ and $\langle\langle\mu\rangle\rangle n_{Q_{1}}$ are isometric, and a direct computation then shows that $\varphi-\langle\mu\rangle \varphi^{\prime}=\langle\langle\alpha \beta \delta, \mu, \alpha, \beta\rangle$, so that

$$
e_{4}\left(\sigma / \sigma^{\prime}\right)=(\alpha \beta \delta, \mu, \alpha, \beta)=(\delta, \mu) \cdot\left[Q_{1}\right] \in H^{4}(F) / E_{4} .
$$

By the common slot Lemma [2, Lemma 1.7], we may find $\nu \in F^{\times}$such that

$$
\left(\delta \delta^{\prime} \mu\right) \cdot\left[Q_{2}\right]=(\nu) \cdot\left[Q_{2}\right]=(\nu) \cdot\left[Q_{1}\right]=(\mu) \cdot\left[Q_{1}\right] .
$$

In particular, we have $(\nu) \cdot[A]=(\nu) \cdot\left[Q_{1}\right]+(\nu) \cdot\left[Q_{2}\right]=0$, and the forms $\langle\langle\nu\rangle\rangle n_{Q_{1}}$ and $\langle\langle\nu\rangle\rangle n_{Q_{2}}$ are isometric. Hence, we have $\langle\langle\nu\rangle\rangle \varphi=\langle\langle\alpha \beta \delta, \nu, \alpha, \beta\rangle\rangle$, so that

$$
\left.(\alpha \beta \delta, \nu, \alpha, \beta)=e_{4}(\langle\nu\rangle\rangle \varphi\right) \in E_{4},
$$

which proves $e_{4}\left(\sigma / \sigma^{\prime}\right)=0$. Alternately, one may also directly check that $\varphi-\langle\mu \nu\rangle \varphi^{\prime}$ is hyperbolic, so that $\varphi$ and $\varphi^{\prime}$ are similar and $\sigma \cong \sigma^{\prime}$.

\section{EXAMPLES OF NONISOMORPHIC INVOLUTIONS WITH TRIVIAL RELATIVE ARASON INVARIANT}

In this section, we construct examples of orthogonal involutions on central simple algebras of degree 8 that are not isomorphic in spite of the fact that their relative Arason invariant is trivial. (In particular, in view of Theorem 2.1, they become isomorphic over any splitting field of the algebra.) By Corollary 4.3 below, such examples do not occur if the index of the algebra is 2. We give two (related) examples: in Example 3.2 the algebra has index 4, and in Example 3.3 it has index 8. The construction below is directly inspired by Hoffmann's examples of nonsimilar half-neighbors in $[12, \S 4]$.

Our first observation is a general result of independent interest:

Lemma 3.1. Let $\sigma$ and $\sigma^{\prime}$ be involutions (of any type) on a central simple $F$-algebra $A$. If $\sigma$ and $\sigma^{\prime}$ are isomorphic after scalar extension to some purely transcendental extension of $F$, then they are isomorphic.

Proof. Suppose first that $A$ is split. If $\sigma$ and $\sigma^{\prime}$ are symplectic, there is nothing to prove since all the symplectic involutions on a split algebra are isomorphic. If $\sigma$ and $\sigma^{\prime}$ are orthogonal or unitary, the lemma is equivalent to the statement that two quadratic or hermitian forms over $F$ are similar if they are similar after scalar extension to a purely transcendental extension. This is easily proved for quadratic forms, see [12, Corollary 3.2], and the hermitian case reduces to the case of quadratic forms by associating to each hermitian form its trace form, which is a quadratic form. For the rest of the proof we may thus assume $A$ is not split; in particular, $F$ is infinite.

Arguing by induction on the transcendence degree, it suffices to prove the lemma when $E=F(x)$ for some indeterminate $x$. Suppose there is an $E$-isomorphism $(A, \sigma)_{E} \cong\left(A, \sigma^{\prime}\right)_{E}$. Then $\sigma$ and $\sigma^{\prime}$ are of the same type, so $\sigma^{\prime}=\operatorname{Int}(s) \circ \sigma$ for some $s \in A^{\times}$such that $\sigma(s)=s$. If the $E$-isomorphism is the inner automorphism $\operatorname{Int}(g)$ of $A_{E}$ for some $g \in A_{E}^{\times}$, then

$$
\operatorname{Int}(g) \circ \sigma=\sigma^{\prime} \circ \operatorname{Int}(g)=\operatorname{Int}\left(s \sigma(g)^{-1}\right) \circ \sigma,
$$

hence $\operatorname{Int}(g)=\operatorname{Int}\left(s \sigma(g)^{-1}\right)$, which means there is an element $e \in E^{\times}$such that

$$
e s=g \sigma(g) \text {. }
$$


Multiplying each side by a suitable factor of the form $f \sigma(f)$ with $f \in F[x]$, we may assume $e \in F[x]$, hence $e s \in A \otimes_{F} F[x]$. By the analogue for involutions of the Cassels-Pfister theorem (see [30]), we may also assume $g \in A \otimes_{F} F[x]$. Let $x_{0} \in F$ be an element that is not a root of $e$. Substituting $x_{0}$ for the indeterminate in $(3)$, we get $g\left(x_{0}\right) \in A$ and $e\left(x_{0}\right) \in F^{\times}$such that

$$
e\left(x_{0}\right) s=g\left(x_{0}\right) \cdot \sigma\left(g\left(x_{0}\right)\right) .
$$

This equation shows that $g\left(x_{0}\right)$ is invertible since $e\left(x_{0}\right) s \in A^{\times}$, and it follows that $\operatorname{Int}\left(g\left(x_{0}\right)\right)$ is an isomorphism between $(A, \sigma)$ and $\left(A, \sigma^{\prime}\right)$.

We now turn to the construction of the examples mentioned at the beginning of this section. The starting point is a field $k$ and a central simple $k$-algebra $E$ with the following properties: $E$ has degree 8 and exponent 2, and it contains a triquadratic field extension $M=k(\sqrt{a}, \sqrt{b}, \sqrt{c})$ but does not have any tensor product decomposition into quaternion algebras of the form

$$
E=(a, u)_{k} \otimes_{k}(b, v)_{k} \otimes_{k}(c, w)_{k} \quad \text { for any } u, v, w \in k^{\times} .
$$

For example, $E$ can be any indecomposable division $k$-algebra of degree 8 and exponent 2, as those constructed by Karpenko in [17, 3.1], since a theorem of Rowen [15, V,Thm.5.6.10] shows that any such algebra contains a triquadratic extension of the center. On the other hand, examples of algebras $E$ as above were used to construct indecomposable division algebras of degree 8 and exponent 2 (over larger fields) in [1] (see also [29]). They exist over rational function fields in one variable over $\mathbb{Q}$ or in two variables over $\mathbb{C}($ see $[8, \S 5])$.

Fix a $k$-algebra $E$ as above, and let $K=k(\sqrt{a})$, viewed as a subfield of $M \subset E$. The centralizer $C_{E} K$ of $K$ in $E$ is a $K$-algebra of degree 4 and exponent 2 containing $M=K(\sqrt{b}, \sqrt{c})$ as a maximal subfield, hence it has a decomposition

$$
C_{E} K=(b, r)_{K} \otimes_{K}(c, s)_{K} \quad \text { for some } r, s \in K^{\times} .
$$

It follows from the projection formula that its corestriction to $k$ is Brauer-equivalent to

$$
\left(b, N_{K / k}(r)\right)_{k} \otimes_{k}\left(c, N_{K / k}(s)\right)_{k} .
$$

On the other hand, $C_{E} K$ is Brauer-equivalent to $E \otimes_{k} K$, and $N_{K / k}\left(E \otimes_{k} K\right)=$ $E^{\otimes 2}=0$ in the Brauer group of $k$, hence

$$
\left(b, N_{K / k}(r)\right)+\left(c, N_{K / k}(s)\right)=0 \quad \text { in } H^{2}(k) .
$$

Example 3.2. Our first example is over the field $F_{1}=k(x, y)$ of rational functions in two variables over $k$. We consider the tensor product of quaternion algebras

$$
A_{1}=(a, x)_{F_{1}} \otimes_{F_{1}}(b, y)_{F_{1}} \otimes_{F_{1}}(c, 1)_{F_{1}} .
$$

The algebra $(c, 1)_{F_{1}}$ is split. Since $x$ and $y$ are indeterminates over $k$ it is easy to see that $(a, x)_{F_{1}} \otimes_{F_{1}}(b, y)_{F_{1}}$ is a division algebra, hence the index of $A_{1}$ is 4 . Let $1, i_{1}, j_{1}$, $i_{1} j_{1}$ (resp. $1, i_{2}, j_{2}, i_{2} j_{2}$, resp. $1, i_{3}, j_{3}, i_{3} j_{3}$ ) denote the standard quaternion basis of the first (resp. second, resp. third) factor. Consider the orthogonal involutions $\rho_{1}=\operatorname{Int}\left(j_{1}\right) \circ-$ on $(a, x)_{F_{1}}, \tau_{1}=\operatorname{Int}\left(j_{2}\right) \circ-$ on $(b, y)_{F_{1}}$, and $\theta_{1}=\operatorname{Int}\left(i_{3}\right) \circ-$ on $(c, 1)_{F_{1}}\left(\right.$ so $\left.\left((c, 1)_{F_{1}}, \theta_{1}\right) \cong \operatorname{Ad}\langle\langle c\rangle\rangle\right)$. Let

$$
\sigma_{1}=\rho_{1} \otimes \tau_{1} \otimes \theta_{1} \quad \text { and } \quad \sigma_{1}^{\prime}=\left(\operatorname{Int}(s) \circ \rho_{1}\right) \otimes \tau_{1} \otimes \theta_{1},
$$


where $s$ is defined in (5) and is identified with an element of $k\left(i_{1}\right)$. The involutions $\rho_{1}, \operatorname{Int}(s) \circ \rho_{1}, \tau_{1}$, and $\theta_{1}$ have discriminant respectively equal to $x, N_{K / k}(s) x, y$ and $c$ modulo $F^{\times 2}$. Applying (1), one may check that

$$
\left((b, y)_{F_{1}}, \tau_{1}\right) \otimes\left((c, 1)_{F_{1}}, \theta_{1}\right) \cong\left((y, c)_{F_{1}},-\right) \otimes\left((y, b c)_{F_{1}},-\right) .
$$

Hence, we may apply Example 1.7 with $\alpha=y, \beta=b c, \gamma=a, \delta=x, \delta^{\prime}=N_{K / k}(s) x$, $\varepsilon=y$, and $\zeta=c$, and we get

and

$$
\begin{gathered}
\varphi_{1}=\langle\langle y, b c\rangle\rangle-\langle b c y\rangle\langle\langle a, x\rangle\rangle-\langle b c x y\rangle\langle\langle y, c\rangle\rangle, \\
\varphi_{1}^{\prime}=\langle\langle y, b c\rangle\rangle-\langle b c y\rangle\left\langle\left\langle a, N_{K / k}(s) x\right\rangle\right\rangle-\left\langle b c N_{K / k}(s) x y\right\rangle\langle\langle y, c\rangle\rangle,
\end{gathered}
$$

$$
e_{3}\left(\sigma_{1} / \sigma_{1}^{\prime}\right)=\left(N_{K / k}(s), y, c\right) \quad \text { in } M_{A}^{3}\left(F_{1}\right) .
$$

By (6) we have $\left(N_{K / k}(s), c\right)=\left(N_{K / k}(r), b\right)$. Since $\left(N_{K / k}(r), a\right)=(c, 1)=0$, it follows that

$$
\left(N_{K / k}(s), y, c\right)=\left(N_{K / k}(r)\right) \cdot((a, x)+(b, y)+(c, 1))=\left(N_{K / k}(r)\right) \cdot\left[A_{1}\right],
$$
so $e_{3}\left(\sigma_{1} / \sigma_{1}^{\prime}\right)=0$.

To compute $e_{4}\left(\sigma_{1} / \sigma_{1}^{\prime}\right)$, observe that we have

$$
\varphi_{1}-\left\langle N_{K / k}(r)\right\rangle \varphi_{1}^{\prime}=\left\langle\left\langle N_{K / k}(r), y, b c\right\rangle\right\rangle-\langle b c x y\rangle\left\langle\left\langle N_{K / k}(r s), y, c\right\rangle\right\rangle .
$$

Now, (6) yields $\left(b c, N_{K / k}(r)\right)=\left(c, N_{K / k}(r s)\right)$ in $H^{2}(k)$, hence

$$
\left\langle\left\langle b c, N_{K / k}(r)\right\rangle\right\rangle=\left\langle\left\langle c, N_{K / k}(r s)\right\rangle\right\rangle .
$$

Substituting in (7), we obtain $\varphi_{1}-\left\langle N_{K / k}(r)\right\rangle \varphi_{1}^{\prime}=\left\langle\left\langle N_{K / k}(r), y, b c, b c x y\right\rangle\right\rangle$. Since $(y, b c, b c y)=0$ in $H^{3}\left(F_{1}\right)$, we finally obtain

$$
e_{4}\left(\sigma_{1} / \sigma_{1}^{\prime}\right)=e_{4}\left(\varphi_{1}-\left\langle N_{K / k}(r)\right\rangle \varphi_{1}^{\prime}\right)=\left(N_{K / k}(r), b c, x, y\right) \quad \text { in } H^{1}\left(F_{1}\right) / E_{4} .
$$

To prove that $\sigma_{1}$ and $\sigma_{1}^{\prime}$ are not isomorphic, we show that $e_{4}\left(\sigma_{1} / \sigma_{1}^{\prime}\right) \neq 0$. Suppose the contrary; we may then find $\nu \in F_{1}^{\times}$such that $(\nu) \cdot[A]=0$ and

$$
\left(N_{K / k}(r), b c, x, y\right)=e_{4}\left(\langle\langle\nu\rangle\rangle \varphi_{1}\right) .
$$

The condition $(\nu) \cdot[A]=0$ is equivalent to

$$
(\nu, a, x)=(\nu, b, y) \quad \text { in } H^{3}\left(F_{1}\right) .
$$

Assuming this condition holds, we have $\langle\langle\nu, a, x\rangle\rangle=\langle\langle\nu, b, y\rangle\rangle$, hence

$$
\langle\langle\nu\rangle\rangle \varphi_{1}=\langle\langle\nu, y, b c\rangle\rangle-\langle b c y\rangle\langle\langle\nu, b, y\rangle\rangle-\langle b c x y\rangle\langle\langle\nu, y, c\rangle\rangle .
$$

Decomposing $\langle\langle\nu, y, b c\rangle\rangle=\langle\langle\nu, b, y\rangle\rangle+\langle b\rangle\langle\langle\nu, y, c\rangle\rangle$, we obtain

$$
\left\langle\langle\nu\rangle \varphi_{1}=\langle\langle b c y, \nu, b, y\rangle\rangle+\langle b\rangle\langle\langle c x y, \nu, y, c\rangle\rangle\right.
$$

hence

$$
e_{4}\left(\left\langle\langle\nu\rangle \varphi_{1}\right)=(b c y, \nu, b, y)+(c x y, \nu, y, c) .\right.
$$

Since $(b y, b, y)=(c y, y, c)=0$, we finally get

$$
e_{4}\left(\left\langle\langle\nu\rangle \varphi_{1}\right)=(c, \nu, b x, y)\right. \text {. }
$$

Thus, we need to derive a contradiction from (8) and

$$
\left(N_{K / k}(r), b c, x, y\right)=(c, \nu, b x, y) \quad \text { in } H^{4}\left(F_{1}\right) .
$$

Extend scalars to $\widehat{F}_{1}=k((x))((y))$. By [20, Cor. VI.1.3], the square classes in $\widehat{F}_{1}$ are represented by elements of the form $u x^{m} y^{n}$ where $u \in k^{\times}$and $m, n=0$ or 1 ; we may thus assume $\nu=\nu_{0} x^{m} y^{n}$ for some $\nu_{0} \in k^{\times}$and some $m, n=0$ or 1 . 
We use the residue maps with respect to the $y$-adic valuation on $\widehat{F}_{1}$ and the $x$-adic valuation on $k((x))$ (see [10, p. 18]),

$$
H^{3}\left(\widehat{F}_{1}\right) \stackrel{\partial_{y}}{\longrightarrow} H^{2}(k((x))) \stackrel{\partial_{x}}{\longrightarrow} H^{1}(k) .
$$

If $n=1$, the image of (8) under the composition $\partial_{x} \circ \partial_{y}$ is

$$
(a)= \begin{cases}0 & \text { if } m=0 \\ (b) & \text { if } m=1\end{cases}
$$

Each case contradicts the fact that $M=k(\sqrt{a}, \sqrt{b}, \sqrt{c})$ is a triquadratic field extension. Hence $n=0$, and we may assume $\nu \in k((x))$. The image of (8) under $\partial_{y}$ is $(\nu, b)=0$, therefore,

$$
(\nu, a, x)=0 \text { in } H^{3}\left(\widehat{F}_{1}\right) \text { and } \quad(\nu, b)=0 \text { in } H^{2}(k((x))) .
$$

Since the map $H^{3}(k((x))) \rightarrow H^{3}\left(\widehat{F}_{1}\right)$ induced by the inclusion $k((x)) \subset \widehat{F}_{1}$ is injective, it follows that the first equation also holds in $H^{3}(k((x)))$. If $m=1$, the image of the second equation under $\partial_{x}$ yields $(b)=0$ in $H^{1}(k)$, a contradiction. Therefore, $\nu=\nu_{0} \in k^{\times}$and the image of the first equation under $\partial_{x}$ yields $\left(\nu_{0}, a\right)=$ 0 in $H^{2}(k)$. Since the map $H^{2}(k) \rightarrow H^{2}(k((x)))$ is injective, we thus have

$$
\left(\nu_{0}, a\right)=\left(\nu_{0}, b\right)=0 \quad \text { in } H^{2}(k) .
$$

On the other hand, taking the image of (9) under $\partial_{x} \circ \partial_{y}$ yields

$$
\left(N_{K / k}(r), b c\right)=\left(c, \nu_{0}\right) \quad \text { in } H^{2}(k) .
$$

Since $\left(\nu_{0}, b\right)=0$, it follows that

$$
\left(N_{K / k}(r) \nu_{0}, b c\right)=0 \quad \text { in } H^{2}(k) .
$$

In view of $(6)$, we also have

$$
\left(N_{K / k}(r s) \nu_{0}, c\right)=0 \quad \text { in } H^{2}(k) .
$$

Since $\left(\nu_{0}, a\right)=0$, we may find $t \in K^{\times}$such that $\nu_{0}=N_{K / k}(t)$ and rewrite $(10)$, (11), and (12) as

$$
\left(N_{K / k}(t), b\right)=\left(N_{K / k}(r t), b c\right)=\left(N_{K / k}(r s t), c\right)=0 \quad \text { in } H^{2}(k) .
$$

Quaternion $K$-algebras whose corestriction is trivial descent to $k$, by [29, Lemma 2.6]. Therefore, we may find $u_{1}, u_{2}, u_{3} \in F^{\times}$such that

$$
(t, b)_{K}=\left(u_{1}, b\right)_{k} \otimes_{k} K, \quad(r t, b c)_{K}=\left(u_{2}, b c\right)_{k} \otimes_{k} K, \quad(r s t, c)_{K}=\left(u_{3}, c\right)_{k} \otimes_{k} K .
$$

Since the cup-product is bilinear, we have

$$
(b, r)_{K}+(c, s)_{K}=(b, t)_{K}+(b c, r t)_{K}+(c, r s t)_{K} \quad \text { in } H^{2}(K)
$$

and it follows that

$$
(b, r)_{K} \otimes_{K}(c, s)_{K}=\left(b, u_{1} u_{2}\right)_{k} \otimes_{k}\left(c, u_{2} u_{3}\right)_{k} \otimes_{k} K .
$$

Therefore, by (5), the centralizer $C_{E} K$ contains a $k$-algebra isomorphic to $\left(b, u_{1} u_{2}\right)_{k} \otimes_{k}$ $\left(c, u_{2} u_{3}\right)_{k}$. The centralizer of this $k$-algebra in $E$ is a quaternion algebra containing $K$, hence $E$ has a decomposition

$$
E=\left(a, u_{4}\right)_{k} \otimes_{k}\left(b, u_{1} u_{2}\right)_{k} \otimes_{k}\left(c, u_{2} u_{3}\right)_{k} \quad \text { for some } u_{4} \in k^{\times} .
$$

This contradicts our hypothesis on $E$ (see (4)) and completes the proof that $e_{4}\left(\sigma_{1} / \sigma_{1}^{\prime}\right)$ is nonzero. 
Example 3.3. Continuing with the notation in Example 3.2, let $z$ be an indeterminate over $F_{1}$ and let $F_{2}$ be the field of functions on the product of the projective quadrics over $F_{1}(z)$ given by the quadratic forms

$$
\left\langle\left\langle c, z, N_{K / k}(r)\right\rangle \quad \text { and } \quad\left\langle\left\langle c, z, N_{K / k}(s)\right\rangle\right\rangle\right. \text {. }
$$

These quadratic forms are isotropic over $F_{1}(z)(\sqrt{z})$, hence $F_{2}(\sqrt{z})$ is purely transcendental over $F_{1}(z)(\sqrt{z})$, and also over $F_{1}$. Let

$$
D=(a, x)_{F_{1}(z)} \otimes_{F_{1}(z)}(b, y)_{F_{1}(z)} \otimes_{F_{1}(z)}(c, z)_{F_{1}(z)} \quad \text { and } \quad A_{2}=D \otimes_{F_{1}(z)} F_{2} .
$$

The algebra $D$ is a division algebra since $x, y, z$ are indeterminates over $k$. The quadratic forms in (13) are in $I^{3} F_{1}(z)$. Therefore, it follows from Merkurjev's index reduction formula $[9,30.11]$ that any $F_{1}(z)$ division algebra remains division over $F_{2}$; in particular, $A_{2}$ is a division algebra. Note that since $F_{2}(\sqrt{z})$ splits $(c, z)_{F_{2}}$ we have an obvious isomorphism

$$
A_{2} \otimes_{F_{2}} F_{2}(\sqrt{z}) \cong A_{1} \otimes_{F_{1}} F_{2}(\sqrt{z}) .
$$

Consider the orthogonal involutions $\rho_{2}=\operatorname{Int}\left(j_{1}\right) \circ-$ on $(a, x)_{F_{2}}, \tau_{2}=\operatorname{Int}\left(j_{2}\right) \circ-$ on $(b, y)_{F_{2}}$, and $\theta_{2}=\operatorname{Int}\left(i_{3}\right) \circ-$ on $(c, z)_{F_{2}}$, and define the following involutions on $A_{2}$ :

$$
\sigma_{2}=\rho_{2} \otimes \tau_{2} \otimes \theta_{2} \quad \text { and } \quad \sigma_{2}^{\prime}=\left(\operatorname{Int}(s) \circ \rho_{2}\right) \otimes \tau_{2} \otimes \theta_{2} .
$$

Clearly, the isomorphism (14) carries $\sigma_{2}$ to $\sigma_{1}$ and $\sigma_{2}^{\prime}$ to $\sigma_{1}^{\prime}$, that is

$$
\left(A_{2}, \sigma_{2}\right)_{F_{2}(\sqrt{z})} \cong\left(A_{1}, \sigma_{1}\right)_{F_{2}(\sqrt{z})} \quad \text { and } \quad\left(A_{2}, \sigma_{2}^{\prime}\right)_{F_{2}(\sqrt{z})} \cong\left(A_{1}, \sigma_{1}^{\prime}\right)_{F_{2}(\sqrt{z})} .
$$

Since $F_{2}(\sqrt{z})$ is purely transcendental over $F_{1}$, and since $\sigma_{1}$ and $\sigma_{1}^{\prime}$ are not isomorphic, it follows from Lemma 3.1 that $\sigma_{2}$ and $\sigma_{2}^{\prime}$ are not isomorphic. Yet, we claim that $e_{3}\left(\sigma_{2} / \sigma_{2}^{\prime}\right)=0$. Again, this relative invariant can be computed by (1) and Example 1.7. The involutions $\tau_{2}$ and $\theta_{2}$ have respective discriminant $y$ and $c$ modulo $F^{\times 2}$, so the components of the Clifford algebra of $\tau_{2} \otimes \theta_{2}$ have Brauer class $(b c, y)$ and $(c, y z)$. Therefore,

$$
e_{3}\left(\sigma_{2} / \sigma_{2}^{\prime}\right)=\left(N_{K / k}(s), b c, y\right)=\left(N_{K / k}(s), c, y z\right) \text { in } M_{A_{2}}^{3}\left(F_{2}\right) .
$$

Now, we have $\left(N_{K / k}(s), c\right)=\left(N_{K / k}(r), b\right)$ by $(6)$, and

$$
\left(N_{K / k}(s), c, z\right)=\left(N_{K / k}(r), c, z\right)=0 \quad \text { in } H^{3}\left(F_{2}\right)
$$

since the quadratic forms (13) split over $F_{2}$. Hence in $H^{3}\left(F_{2}\right)$, we have

$$
\begin{gathered}
\left(N_{K / k}(s), c, y z\right)=\left(N_{K / k}(s), c, y\right)=\left(N_{K / k}(r), b, y\right), \text { and } \\
\left(N_{K / k}(r), b, y\right)=\left(N_{K / k}(r)\right) \cdot((a, x)+(b, y)+(c, z))=\left(N_{K / k}(r)\right) \cdot\left[A_{2}\right] .
\end{gathered}
$$

Therefore, $e_{3}\left(\sigma_{2} / \sigma_{2}^{\prime}\right)=0$.

\section{Algebras of index Dividing 4}

Throughout this section, we assume that the algebra $A$ has index at most 4 . Thus, $A \cong \operatorname{End}_{D} V$ for some central division $F$-algebra $D$ of exponent 2 and some right $D$-vector space $V$ of dimension 2, 4, or 8 . It follows that $A$ carries orthogonal hyperbolic involutions, which are those that are isomorphic to involutions on $\operatorname{End}_{D} V$ adjoint to hyperbolic hermitian forms on $V$ (with respect to a given orthogonal involution on $D$ ). Since all hyperbolic hermitian forms on $V$ are isometric, all the orthogonal hyperbolic involutions on $A$ are isomorphic. They are totally decomposable since they have trivial discriminant and Clifford invariant. Fixing an 
arbitrary orthogonal hyperbolic involution $\sigma_{0}$ on $A$, we define the Arason invariant of the totally decomposable orthogonal involution $\sigma$ on $A$ by

$$
e_{3}(\sigma)=e_{3}\left(\sigma / \sigma_{0}\right) \in M_{A}^{3}(F)
$$

In particular, if $\sigma$ is hyperbolic, we have $e_{3}(\sigma)=e_{3}\left(\sigma / \sigma_{0}\right)=0$ since $\sigma$ and $\sigma_{0}$ are isomorphic. Hence, it follows from (2) that $e_{3}(\sigma) \in M_{A}^{3}(F)$ does not depend on the particular choice we made for $\sigma_{0}$. Moreover for any totally decomposable orthogonal involutions $\sigma, \sigma^{\prime}$ on $A$ their relative Arason invariant is given by

$$
e_{3}\left(\sigma / \sigma^{\prime}\right)=e_{3}(\sigma)+e_{3}\left(\sigma^{\prime}\right)=e_{3}(\sigma)-e_{3}\left(\sigma^{\prime}\right)
$$

As an easy consequence of Theorem 2.1, we now prove that the Arason invariant does detect hyperbolicity:

Corollary 4.1. The totally decomposable involution $\sigma$ is hyperbolic if and only if $e_{3}(\sigma)=0$.

Proof. We have already explained that $e_{3}(\sigma)$ is zero if $\sigma$ is hyperbolic. To prove the converse, let us pick an arbitrary hyperbolic involution $\sigma_{0}$ on $A$, and consider quadratic forms $\varphi$ and $\varphi_{0}$ associated respectively to $\sigma$ and $\sigma_{0}$ by triality. If $e_{3}(\sigma)=$ $e_{3}\left(\sigma / \sigma_{0}\right)$ is trivial, Theorem 2.1 provides $\mu \in F^{\times}$such that $\varphi+\langle\mu\rangle \varphi_{0}$ is similar to a 4 -fold Pfister form. As explained in $[25,5.1]$, since $\sigma_{0}$ is hyperbolic, the quadratic form $\varphi_{0}$ is isotropic. Hence $\varphi+\langle\mu\rangle \varphi_{0}$ is hyperbolic, $\varphi$ and $\varphi_{0}$ are similar, and $\sigma$ and $\sigma_{0}$ are isomorphic. Thus $\sigma$ is hyperbolic.

If $A$ is split, and $\sigma$ is adjoint to the 3-fold Pfister form $\pi$, it follows from 1.5 that its Arason invariant is given by

$$
e_{3}(\sigma)=e_{3}(\pi) \text { in } H^{3}(F) .
$$

In particular, $e_{3}(\sigma)$ is always represented by a single symbol of $H^{3}(F)$. In the next two sections, we will show that this remains true for algebras of index 2 , but not in index 4.

4.1. Algebras of index 2. Let us first assume that $A$ has index 2, and pick a quaternion algebra $Q$ Brauer-equivalent to $A$. As we already mentioned, the Arason invariant is classifying in this case. More precisely, we have:

Theorem 4.2. Let $\sigma$ be a totally decomposable orthogonal involution on the algebra $A=M_{4}(Q)$. There exist $\lambda, \mu \in F^{\times}$and an orthogonal involution $\rho$ on $Q$ such that

$$
(A, \sigma) \cong \operatorname{Ad}_{\langle\langle\lambda, \mu\rangle\rangle} \otimes_{F}(Q, \rho) \text {. }
$$

When this isomorphism holds, we have

$$
e_{3}(\sigma)=(\lambda, \mu) \cdot d(\rho) \quad \text { in } M_{Q}^{3}(F) .
$$

Conversely, if $e_{3}(\sigma)=(\lambda, \mu, \delta)$ in $M_{Q}^{3}(F)$ for some $\lambda, \mu, \delta \in F^{\times}$such that $F(\sqrt{\delta})$ splits $A$, then (15) holds for some orthogonal involution $\rho$ on $Q$ with $d(\rho)=(\delta)$.

Proof. The existence of a decomposition of the form (15) was proved by Becher [5, Thm. 2]. Starting from there, we may find $\delta$ and $\gamma \in F^{\times}$so that $d(\rho)=(\delta)$ and $[Q]=(\gamma, \delta)$. Applying $(1)$ to $\operatorname{Ad}_{\langle\langle\mu\rangle\rangle} \otimes(Q, \rho)$, we get

$$
(A, \sigma)=\operatorname{Ad}_{\langle\langle\lambda\rangle\rangle} \otimes\left((\mu, \delta)_{F},-\right) \otimes\left((\mu \gamma, \delta)_{F},-\right) .
$$

Since the involution $\sigma_{0}=\operatorname{ad}_{\langle\langle 1\rangle\rangle} \otimes-\otimes-$ is hyperbolic, Example 1.7 gives

$$
e_{3}(\sigma)=(\lambda) \cdot(\mu, \delta)=(\lambda, \mu) \cdot d(\rho) \in M_{A}^{3}(F) .
$$


Conversely, suppose $e_{3}(\sigma)=(\lambda, \mu, \delta)$ in $H^{3}(F) /\left(F^{\times} \cdot[Q]\right)$ for some $\lambda, \mu, \delta \in F^{\times}$ such that $F(\sqrt{\delta})$ splits $A$. Since $e_{3}(\sigma)$ splits over $F(\sqrt{\delta})$, Corollary 4.1 shows that $\sigma$ becomes hyperbolic over $F(\sqrt{\delta})$. Using either the proposition in the Appendix of $[24]$, or $[3,3.4(2)]$, one may easily check that $(A, \sigma)$ decomposes as

$$
(A, \sigma) \cong \operatorname{Ad}_{q} \otimes_{F}(Q, \rho)
$$

for some 4-dimensional quadratic form $q$ over $F$ and some orthogonal involution $\rho$ on $Q$ with $d(\rho)=(\delta)$. Since $(Q, \rho)_{F_{Q}} \cong \operatorname{Ad}_{\langle\langle\delta\rangle\rangle}$, we have

$$
(A, \sigma)_{F_{Q}} \cong \operatorname{Ad}_{q \otimes\langle\langle\delta\rangle\rangle}
$$

On the other hand, $e_{3}(\sigma)$ is represented by $(\lambda, \mu, \delta)$. Hence $(A, \sigma)_{F_{Q}} \cong \operatorname{Ad}_{\langle\langle\lambda, \mu, \delta\rangle\rangle}$, so that the forms $q \otimes\langle\langle\delta\rangle\rangle$ and $\langle\langle\lambda, \mu, \delta\rangle\rangle$ are similar over $F_{Q}$. To deduce a decomposition of the type (15), we use the same argument as in the proof of [5, Thm. 2]. Scaling if necessary, we may assume the form $q$ represents 1 . Since $\langle\langle\lambda, \mu, \delta\rangle\rangle$ is a Pfister form, the similarity then has to be an isometry, so that $(\langle\langle\lambda, \mu\rangle\rangle-q)\langle\langle\delta\rangle\rangle=0$ in the Witt group of $F_{Q}$. This precisely means that the hermitian form $(\langle\langle\lambda, \mu\rangle\rangle-q) \cdot\langle 1\rangle$ over $(Q, \rho)$ is in the kernel of the scalar extension map of Witt groups $W(Q, \rho) \rightarrow$ $W\left(F_{Q}\right)$. But this map is injective by [7] or [22, 3.3], hence the hermitian forms $\langle\langle\lambda, \mu\rangle\rangle \cdot\langle 1\rangle$ and $q \cdot\langle 1\rangle$ over $(Q, \rho)$ are isometric. Therefore,

$$
\operatorname{Ad}_{q} \otimes_{F}(Q, \rho) \cong \operatorname{Ad}_{\langle\langle\lambda, \mu\rangle\rangle} \otimes_{F}(Q, \rho),
$$

proving (15).

From this we get:

Corollary 4.3. Let $A$ be a central simple F-algebra of degree 8 and index 2, endowed with totally decomposable orthogonal involutions $\sigma$ and $\sigma^{\prime}$.

(1) The Arason invariant $e_{3}(\sigma)$ is represented in $H^{3}(F) /\left(F^{\times} \cdot[A]\right)$ by a single 3-symbol in $H^{3}(F)$.

(2) The involutions $\sigma$ and $\sigma^{\prime}$ are isomorphic if and only if $e_{3}(\sigma)=e_{3}\left(\sigma^{\prime}\right)$.

Proof. It readily follows from Theorem 4.2 that $e_{3}(\sigma)$ is represented by a single symbol. If $\sigma$ and $\sigma^{\prime}$ are totally decomposable orthogonal involutions such that $e_{3}(\sigma)=e_{3}\left(\sigma^{\prime}\right)$, fix $\lambda, \mu, \delta \in F^{\times}$such that $F(\sqrt{\delta})$ splits $A$ and

$$
e_{3}(\sigma)=e_{3}\left(\sigma^{\prime}\right)=(\lambda, \mu, \delta) \text { in } M_{Q}^{3}(F) .
$$

By Theorem 4.2 we have

$$
(A, \sigma) \cong \operatorname{Ad}_{\langle\langle\lambda, \mu\rangle\rangle} \otimes_{F}(Q, \rho) \cong\left(A, \sigma^{\prime}\right)
$$

for some orthogonal involution $\rho$ on $Q$ with $d(\rho)=(\delta)$, hence $\sigma$ and $\sigma^{\prime}$ are isomorphic.

Remark 4.4. The arguments developed in this subsection are still valid in higher degree, and enable us to define higher invariants, with values in

$$
M_{A}^{n}(F)=H^{n}(F) / H^{n-2}(F) \cdot[A]
$$

where $H^{n-2}(F) \cdot[A]$ denotes the subgroup $\left\{c \cdot[A], c \in H^{n-2}(F)\right\} \subset H^{n}(F)$. Indeed, let $(A, \sigma)$ be a totally decomposable algebra with orthogonal involution of degree $2^{n}$, Brauer equivalent to a quaternion algebra $Q$. By Becher's theorem, it decomposes as $(A, \sigma)=\operatorname{Ad}_{\pi} \otimes(Q, \rho)$ for some $(n-1)$-fold Pfister form $\pi$ and some orthogonal 
involution $\rho$ on $Q$. Over $F_{Q}$, the involution $\sigma$ is adjoint to the form $\pi \otimes\langle\langle\delta\rangle$, where $(\delta)=d(\rho)$. Hence,

$$
e_{n}(\sigma)=e_{n-1}(\pi) \cdot d(\rho) \in M_{Q}^{n}(F)
$$

is a well defined invariant of $\sigma$. Moreover, again by the very same arguments as in degree 8, two totally decomposable involutions $\sigma$ and $\sigma^{\prime}$ of $A$ are isomorphic if and only if $e_{n}(\sigma)=e_{n}\left(\sigma^{\prime}\right)$.

Alternately, this can also be deduced from Berhuy's Theorem 13 in [6]. Indeed, one may easily check from $[6,2.4]$ that our invariant $e_{n}(\sigma)$ coincides with his $e_{n, Q}(h)$ if $\sigma$ is adjoint to the skew-hermitian form $h$ over $(Q,-)$.

4.2. Algebras of index 4. We now assume that $A$ has index 4 , and let $D$ be a division algebra Brauer equivalent to $A$. As explained in [21, Thm.1.1], for every totally decomposable orthogonal involution $\sigma$, there is a decomposition

$$
(A, \sigma)=\operatorname{Ad}_{\langle\langle\lambda\rangle\rangle} \otimes(D, \theta)
$$

for some $\lambda \in F^{\times}$and some orthogonal involution $\theta$ on $D$. Since $(D, \theta)$ need not be decomposable, we use the following refined decomposition to compute $e_{3}(\sigma)$ :

Theorem 4.5. Let $\sigma$ be a totally decomposable orthogonal involution on the index 4 algebra $A=M_{2}(D)$. There exists a quadratic étale extension $L / F$, a quaternion algebra $Q$ over $L$ and $\mu \in L^{\times}$such that

$$
(A, \sigma) \cong \operatorname{Ad}_{\left\langle\left\langle N_{L / F}(\mu)\right\rangle\right\rangle} \otimes N_{L / F}(Q,-) .
$$

When this isomorphism holds, we have

$$
e_{3}(\sigma)=N_{L / F}((\mu) \cdot[Q]) \quad \text { in } M_{A}^{3}(F) .
$$

Proof. The existence of such a decomposition is explained in $[21, \S 4]$, where we also compute a quadratic form $\varphi$ associated to $\sigma$ by triality. We sketch the argument for the reader's convenience. Consider the quadratic étale extension $L / F$ corresponding to the discriminant $d(\theta) \in F^{\times} / F^{\times 2}$. By $[18,(15.7)]$, the Clifford algebra $\mathcal{C}(D, \theta)$ is a quaternion algebra $Q$ over $L$, and

$$
(D, \theta) \cong N_{L / F}(Q,-)
$$

Hence, it only remains to prove that $\lambda$ is the norm of some $\mu \in L^{\times}$, or equivalently that $(\lambda) \cdot d(\theta)=0 \in H^{2}(F)$. By [28, 4.12] (see also [18, p.150]), $(\lambda) \cdot d(\theta)$ is the Brauer class of one component of the Clifford algebra $\mathcal{C}(A, \sigma)$, while the other is $(\lambda) \cdot d(\theta)+[A]$. Since $\sigma$ is totally decomposable, one of these classes is trivial. As $A$ has index 4 , it has to be the first one, and (16) is proved.

For any quadratic form $\psi$ over $L$, we let $\operatorname{tr}_{\star}(\psi)$ be the transfer of $\psi$ associated to the trace map $\operatorname{tr}: L \rightarrow F$, as defined in [20, VII.1.2]. From [21, Prop.2.1], one may easily check that each component of the Clifford algebra of $\operatorname{tr}_{\star}\left(\langle\delta \mu\rangle n_{Q}\right)$, where $n_{Q}$ denotes the norm form of $Q$, is $\operatorname{Ad}_{\left\langle\left\langle N_{L / F}(\mu)\right\rangle\right\rangle} \otimes N_{L / F}(Q,-) \cong(A, \sigma)$. Hence $\operatorname{tr}_{\star}\left(\langle\delta \mu\rangle n_{Q}\right)$ is a quadratic form associated to $\sigma$ by triality. Moreover, by the same argument, the quadratic form $\varphi_{0}=\operatorname{tr}_{\star}\left(\langle\delta\rangle n_{Q}\right)$ is associated to the hyperbolic involution $\sigma_{0}=\operatorname{ad}\langle\langle 1\rangle\rangle \otimes \theta$. Hence the Arason invariant $e_{3}(\sigma)=e_{3}\left(\sigma / \sigma_{0}\right) \in M_{A}^{3}(F)$ is represented by

$$
e_{3}\left(\varphi-\varphi_{0}\right)=e_{3}\left(\operatorname{tr}_{\star}\left(\langle\delta \mu\rangle\langle\langle\mu\rangle\rangle n_{Q}\right)\right)=N_{L / F}((\mu) \cdot[Q]) \in H^{3}(F) .
$$


Remark 4.6. If the algebra with involution $(A, \sigma)$ decomposes as

$$
(A, \sigma)=\operatorname{Ad}_{\langle\langle\lambda\rangle\rangle} \otimes\left(Q_{1},-\right) \otimes\left(Q_{2},-\right),
$$

then the formula easily follows from Example 1.7. Indeed, since the involution $\sigma_{0}=\langle\langle 1\rangle\rangle \otimes-\otimes-$ is hyperbolic, we have

$$
e_{3}(\sigma)=e_{3}\left(\sigma / \sigma_{0}\right)=(\lambda) \cdot\left[Q_{1}\right] \in M_{A}^{3}(F) .
$$

On the other hand, the decomposition above is a decomposition as in (16), with $L=F \times F, Q=Q_{1} \times Q_{2}$, and $\mu=(\lambda, 1) \in F \times F$, so that $N_{L / F}((\mu) \cdot[Q])=(\lambda) \cdot\left[Q_{1}\right]$.

Example 4.7. By (1), the algebra with involution $\left(A_{1}, \sigma_{1}\right)$ of Example 3.2 decomposes as

$$
\left(A_{1}, \sigma_{1}\right) \cong \operatorname{Ad}_{\langle\langle c\rangle\rangle} \otimes\left(Q_{1},-\right) \otimes\left(Q_{2},-\right),
$$

where

$$
Q_{1}=(a y, x)_{F_{1}} \quad \text { and } \quad Q_{2}=(b x, y)_{F_{1}} .
$$

Hence, the Arason invariant of $\sigma_{1}$ is given by

$$
e_{3}\left(\sigma_{1}\right)=(c) \cdot\left[Q_{1}\right]=(c, a y, x) \in M_{A}^{3}(F) .
$$

Observe that if $(A, \sigma)$ decomposes as in $(17)$, then $e_{3}(\sigma)$ is represented by a single 3-symbol. The following proposition, which is a reformulation of Sivatski's Proposition 2 in [27], shows that the converse also holds:

Proposition 4.8. Let $(A, \sigma)$ be a central simple $F$-algebra of degree 8 and index 4 with totally decomposable orthogonal involution, and let $\varphi \in I^{2} F$ be a quadratic form associated to $\sigma$ by triality. The following are equivalent:

(i) $e_{3}(\sigma)$ can be represented by a single 3 -symbol $(\alpha, \beta, \gamma)$ in $H^{3}(F)$;

(ii) $\varphi \cong \pi_{1} \perp \pi_{2}$ for some quadratic forms $\pi_{1}$, $\pi_{2}$ of dimension 4 with trivial discriminant;

(iii) $(A, \sigma)$ has a decomposition of the form $\operatorname{Ad}_{\langle\langle\lambda\rangle\rangle} \otimes\left(Q_{1},-\right) \otimes\left(Q_{2},-\right)$ for some quaternion $F$-algebras $Q_{1}, Q_{2}$.

We refer the reader to $[14, \S 16],[13]$ and $[27, \S 2]$ for examples either of 8dimensional quadratic forms with trivial discriminant and Clifford algebra of index 4 that do not decompose as in (ii), or totally decomposable degree 8 and index 4 algebras with involution that do not decompose as in (iii). They all prove that in index 4 , the invariant $e_{3}(\sigma)$ is not always represented by a single 3 -symbol.

Remark 4.9. In [25], we constructed examples of algebras with involution that become hyperbolic over the function field of a conic and do not contain the corresponding quaternion algebra endowed with its canonical involution. Another example can be derived from the triple $\left(A_{1}, \sigma_{1}, \sigma_{1}^{\prime}\right)$ of Example 3.2. Indeed, since $e_{3}\left(\sigma_{1} / \sigma_{1}^{\prime}\right)=0$, we have $e_{3}\left(\sigma_{1}\right)=e_{3}\left(\sigma_{1}^{\prime}\right) \in M_{A}^{3}(F)$. This invariant was computed in Example 4.7, and becomes trivial over the field $F_{Q_{1}}$. Hence, by Theorem 4.1, both involutions $\sigma_{1}$ and $\sigma_{1}^{\prime}$ become hyperbolic over $F_{Q_{1}}$. One of them, namely $\left(A_{1}, \sigma_{1}\right)$ does contain $\left(Q_{1},-\right)$ as a subalgebra with involution, by (18). Assume $\left(A_{1}, \sigma_{1}^{\prime}\right)$ also does. Then, the restriction of $\sigma_{1}^{\prime}$ to the centraliser $Q_{2} \otimes M_{2}(F)$ of $Q_{1}$ in $A_{1}$ is adjoint to a 2-dimensional skew hermitian form over $\left(Q_{2},-\right)$, which, after scaling if necesssary, diagonalises as $\left\langle 1,-c^{\prime}\right\rangle$ for some $c^{\prime} \in F^{\times}$. Hence, we get

$$
\left(A_{1}, \sigma_{1}^{\prime}\right) \cong \operatorname{Ad}_{\left\langle\left\langle c^{\prime}\right\rangle\right\rangle} \otimes\left(Q_{1},-\right) \otimes\left(Q_{2},-\right) .
$$


By Proposition 2.7, this implies that $\sigma_{1} \cong \sigma_{1}^{\prime}$. This is impossible since we proved in Example 3.2 that the involutions $\sigma_{1}$ and $\sigma_{1}^{\prime}$ are not isomorphic.

\section{REFERENCES}

[1] S. A. Amitsur, L. H. Rowen and J.-P. Tignol, Division algebras of degree 4 and 8 with involution, Israel J. Math. 33 (1979), no. 2, 133-148.

[2] J. Kr. Arason, Cohomologische invarianten quadratischer Formen, J. Algebra 36 (1975), no. 3, 448-491.

[3] E. Bayer-Fluckiger, D. B. Shapiro and J.-P. Tignol, Hyperbolic involutions, Math. Z. 214 (1993), no. 3, 461-476.

[4] E. Bayer-Fluckiger, R. Parimala and A. Quéguiner-Mathieu, Pfister involutions, Proc. Indian Acad. Sci. Math. Sci. 113 (2003), no. 4, 365-377.

[5] K. J. Becher, A proof of the Pfister factor conjecture, Invent. Math. 173 (2008), no. 1, 1-6.

[6] G. Berhuy, Cohomological invariants of quaternionic skew-Hermitian forms, Arch. Math. (Basel) 88 (2007), no. 5, 434-447.

[7] I. Dejaiffe, Formes antihermitiennes devenant hyperboliques sur un corps de déploiement, C. R. Acad. Sci. Paris Sér. I Math. 332 (2001), no. 2, 105-108.

[8] R. Elman et al., Witt rings and Brauer groups under multiquadratic extensions. I, Amer. J. Math. 105 (1983), no. 5, 1119-1170.

[9] R. Elman, N. Karpenko and A. Merkurjev, The algebraic and geometric theory of quadratic forms, Amer. Math. Soc., Providence, RI, 2008.

[10] S. Garibaldi, A. Merkurjev and J.-P. Serre, Cohomological invariants in Galois cohomology, Amer. Math. Soc., Providence, RI, 2003.

[11] S. Garibaldi, Orthogonal involutions on algebras of degree 16 and the Killing form of $E_{8}$, in Quadratic forms - algebra, arithmetic, and geometry, 131-162, Contemp. Math., 493, Amer. Math. Soc., Providence, RI.

[12] D. W. Hoffmann, Similarity of quadratic forms and half-neighbors, J. Algebra 204 (1998), no. $1,255-280$.

[13] D. W. Hoffmann and J.-P. Tignol, On 14-dimensional quadratic forms in $I^{3}$, 8-dimensional forms in $I^{2}$, and the common value property, Doc. Math. 3 (1998), 189-214 (electronic).

[14] O.T. Izhboldin and N. A. Karpenko, Some new examples in the theory of quadratic forms, Math. Z. 234 (2000), 647-695.

[15] N. Jacobson, Finite-dimensional division algebras over fields, Springer, Berlin, 1996.

[16] B. Kahn, Un invariant de degré 2 des algèbres centrales simples d'exposant 2, preprint.

[17] N. A. Karpenko, Torsion in $\mathrm{CH}^{2}$ of Severi-Brauer varieties and indecomposability of generic algebras, Manuscripta Math. 88 (1995), no. 1, 109-117.

[18] M.-A. Knus et al., The book of involutions, Amer. Math. Soc., Providence, RI, 1998.

[19] A. Laghribi, Isotropie de certaines formes quadratiques de dimensions 7 et 8 sur le corps des fonctions d'une quadrique, Duke Math. J. 85 (1996), no. 2, 397-410.

[20] T. Y. Lam, Introduction to quadratic forms over fields, Amer. Math. Soc., Providence, RI, 2005.

[21] A. Masquelein, A. Quéguiner-Mathieu and J.-P. Tignol, Quadratic forms of dimension 8 with trivial discriminant and Clifford algebra of index 4, Arch. Math. (Basel) 93 (2009), no. 2, 129-138.

[22] R. Parimala, R. Sridharan and V. Suresh, Hermitian analogue of a theorem of Springer, J. Algebra 243 (2001), no. 2, 780-789.

[23] E. Peyre, Galois cohomology in degree three and homogeneous varieties, K-Theory 15 (1998), no. 2, 99-145.

[24] A. Quéguiner-Mathieu and J.-P. Tignol, Discriminant and Clifford algebras, Math. Z. 240 (2002), no. 2, 345-384.

[25] A. Quéguiner-Mathieu and J.-P. Tignol, Algebras with involution that become hyperbolic over the function field of a conic, Israel J. Math., 180 (2010), 317-344.

[26] A. Quéguiner-Mathieu and J.-P. Tignol, Arason invariant for algebras with orthogonal involutions, in preparation.

[27] A. S. Sivatski, Applications of Clifford algebras to involutions and quadratic forms, Comm. Algebra 33 (2005), no. 3, 937-951.

[28] D. Tao, The generalized even Clifford algebra, J. Algebra 172 (1995), no. 1, 184-204. 
[29] J.-P. Tignol, Corps à involution neutralisés par une extension abélienne élémentaire, in The Brauer group (Sem., Les Plans-sur-Bex, 1980), 1-34, Lecture Notes in Math., 844, Springer, Berlin.

[30] J.-P. Tignol, A Cassels-Pfister theorem for involutions on central simple algebras, J. Algebra 181 (1996), no. 3, 857-875.

[31] J.-P. Tignol, Cohomological invariants of central simple algebras with involution, Quadratic forms, linear algebraic groups, and cohomology, 137-171, Dev. Math., 18, Springer, New York, 2010 .

LAGA - UMR 7539 du CNRS, Université Paris 13, F-93430 Villetaneuse, and UPEC, F-94010 Créteil, France

Zukunftskolleg, Universität Konstanz, D-78457 Konstanz, Germany, and ICTEAM, Université Catholique de Louvain, B-1348 Louvain-la-Neuve, Belgium 